\title{
INCIDENCIA Y PREVALENCIA DE LOS FACTORES DE RIESGO EN EL DESARROLLO
}

\author{
Poch Olivé, $M^{a}$ Luisa* ; \\ Ruiz del Prado, $M^{a}$ Yolanda*; \\ Sastre Riba, Sylvia**; \\ Escolano Pérez, Elena** ; \\ Merino Moreno, Natalia** ; \\ *Complejo hospitalario San Millán-San Pedro, Logroño \\ **Universidad de La Rioja
}

\section{RESUMEN}

El objetivo de este estudio es conocer la incidencia y prevalencia de los factores de riesgo en el desarrollo en La Rioja con el fin de compararlos con otros estudios realizados y llegar a establecer las necesidades de intervención temprana en esta Comunidad.

Se han recopilado y analizado los datos correspondientes a la totalidad de los nacimientos ocurridos durante los años 1998 a 2003 (inclusive) en La Rioja. Se ha extraído, mediante el programa SPSS, la incidencia y prevalencia de los factores de riesgo (pre, peri y postnatal), comparativamente entre ellos, así como el momento de detección.

Los resultados muestran: a) una tendencia al alza de la tasa de natalidad; b) prevalencia del riesgo situada en el intervalo entre el $3^{\prime} 51 \%$ y $4^{\prime} 73 \%$; c) entre los factores de riesgo, la prematuridad es la de mayor prevalencia e incidencia; $y$, d) la detección del riesgo se realiza en edad temprana, predominantemente durante los primeros seis meses de vida, con un porcentaje medio de detección neonatal del $71^{\prime} 52 \%$ diferencial interanualmente.

Conocer las situaciones de riesgo y su detección temprana permiten establecer programas de prevención e intervención infantil y familiar para optimizar el desarrollo.

PALABRAS CLAVE: recién nacido de riesgo, desarrollo temprano, factores de riesgo, atención temprana.

[1] Este trabajo ha recibido el apoyo de la Consejería de Salud del Gobierno Autónomo de La Rioja. Expresamos el agradecimiento por su inestimable colaboración a: Dra. C. Garijo; R. Farriol y J. Gil. 


\begin{abstract}
The aim of this study was to evaluate the incidence and prevalence of developmental risk factors in the population of La Rioja, in order to compare these data with other studies and identify the needs of early intervention in the region.

Data on all infant born between January 1998 and December 2003 (inclusive) in La Rioja were included and considered in this study. Risk factors (pre, peri or postnatal) for unfavourable outcomes were studied in this group, as well as the time of diagnosis. Incidence and prevalence data and statistical differences between early or delayed diagnosis were estimated with the SPSS program.

Results show: a) an increase in birthrate; b) risk prevalence was within the $3.51-4.73 \%$ range; c) an increasing rate of preterm births was found in this population; and d) finally, most risk newborns were identified at an early age, mainly in the first six months of life $(71.52 \%$ of them in the neonatal period), with significant year-on-year differences.

The knowledge of risk situations and their early detection allow to establish programs focused on children and families in order to ameliorate developmental courses.
\end{abstract}

KEY WORDS: risk infants, early development, risk factors, early intervention.

\title{
Introducción
}

Los avances actuales en Neonatología y en los cuidados de salud de los recién nacidos, unidos a los cambios socioculturales acontecidos en las últimas décadas han supuesto una reorientación de los conceptos de prevención, atención y protección de la infancia en el amplio marco biopsicosocial (Arizcun, 2002; Blackman, 2002; Grupo de Atención Temprana, 2000; Guralnick, 1993; Sastre y Poch, 2001; Valle Trapero, 1999). Este fenómeno ha estado acompañado de una revisión del concepto de Salud, entendida como el bienestar físico, mental y social del individuo (OMS, 2006), por lo tanto, no sólo como "ausencia de enfermedad".

Por otra parte, la investigación neuropsicológica del desarrollo, ha puesto en evidencia la importancia de las primeras edades para el desarrollo posterior poniendo de manifiesto las necesidades a cubrir durante la primera infancia para potenciar la calidad de vida infantil. En suma, todo ello ha comportado que, progresivamente, aumenten las políticas y acciones dirigidas hacia la detección, cada vez más temprana, de los factores de riesgo biopsicosocial que pueden alterar el bienestar que comporta el concepto actual de Salud.

Paralelamente, la medicina ha desarrollado métodos y técnicas capaces de aumentar la supervivencia de neonatos en edades gestacionales cada vez menores o con otros problemas de salud pre o perinatales. No obstante, estos avances tan deseables y necesarios se han mostrado insuficientes para asegurar el bienestar y la salud de estos niños, si no están acompañados de unas medidas de intervención interdisciplinar coordinadas y bien planificadas, lo cual redunda de nuevo en la 
necesidad de detectar lo más tempranamente posible el riesgo con el fin de prevenir sus posibles efectos negativos.

De ahí, pues, la importancia de: a) delimitar el "riesgo"; b) conocer su incidencia en función del momento en que se produce (prenatal, perinatal o postnatal); y, c) seguir la manifestación de sus posibles secuelas a corto, medio o largo plazo y sus consecuencias en el desarrollo.

Para ello, previamente a las medidas de detección y/o de intervención, es preciso diferenciar lo que es un factor de riesgo, respecto del "niño de riesgo". Por una parte, el factor de riesgo constituye un elemento que se aplica a individuos o grupos de población, entendiendo como tal un acontecimiento negativo o positivo de carácter biológico, emocional y/o social, al que el sujeto ha podido estar expuesto pre-peri o postnatalmente y que puede tener una repercusión negativa en su desarrollo a corto, medio o largo plazo.

Por otra parte, el niño de riesgo, es el niño que, por sus antecedentes pre o perinatales, tiene más posibilidades de presentar problemas de su desarrollo en los diversos ámbitos, pudiendo ser transitorios o permanentes (Grupo de Atención Temprana, 2000).

Por lo tanto, hay que conocer cuáles son los factores de riesgo para determinar quién puede considerarse como niño de riesgo, teniendo en cuenta que, cuánto más temprana es su incidencia, más serio puede ser su pronóstico.

Los mencionados factores de riesgo, en continua revisión, se han ampliado a partir de la investigación realizada en los últimos 30 años, desde el inicial marco biológico hasta contemplar ampliamente situaciones de tipo psicológico y social que pueden intervenir aisladamente o en combinación con los anteriores. Todos ellos, son recogidos extensamente en el Libro Blanco de Atención Temprana (Grupo de Atención Temprana, 2000).

Autores relevantes (Farley, Mason, Rice, Habel, Scribner, and Cohen, 2006; Mellier \& Fessard, 1998; Thompson, Irgens, Rasmussen, Daltveit, 2006; Valle Trapero, 2002; Wolke, 2005) señalan que, los factores de riesgo iniciales, fuertemente saturados en componentes biológicos, tienden a fundirse, a lo largo de los primeros años, con los factores socio-económicos y las condiciones de la interacción del niño con su entorno familiar, son estos factores actuando conjuntamente los que pueden condicionar el desarrollo del niño.

Estos postulados se fundamentan en los resultados obtenidos por la investigación interdisciplinar del desarrollo que han puesto en evidencia, no sólo que el desarroIlo consiste en un proceso continuado de cambio determinado por la estrecha interrelación entre factores neurobiológicos y factores de carácter social (De Haan, \& Johnson, 2003; Diamond, 2002; Karmiloff-Smith, 1997; Munakata, Casey, \& Diamond, 2004; Plomin \& Petrill, 1997; Quartz \& Sejnowski, 1997) sino también, la importancia que tienen los primeros años de vida para el desarrollo posterior del sujeto (Mounoud, 1996). Especialmente, la edad comprendida entre los 18 primeros meses de vida es aquella en la que el ser humano realiza la mayor tasa de ganancias que serán determinantes como base de la consecución de competencias posteriores. De nuevo, se deduce la importancia de realizar la detección de forma temprana.

Estas tesis se acompañan de aquellas que postulan la necesidad de que el desarrollo, entendido como cambio y despliegue sucesivo de competencias, tenga una tónica armónica y organizada, siendo los factores de riesgo un condicionante signi- 
ficativo de disarmonización, con la posible desestructuración y/o deficienciación consecuente (Cambrodí, 1983). Todo ello ha comportado un especial interés hacia el conocimiento de la incidencia de los factores de riesgo a los que ha estado expuesto tempranamente un sujeto, de manera que, los resultados de las investigaciones longitudinales actuales en este campo, indican que numerosos déficits cognitivos y problemas de conducta emergentes en la primera infancia se manifiestan entre niños nacidos con factores de riesgo como: la prematuridad (Elster, 2000; Figueras Aloy, 2004; Lumley, 2003; van Baar, van Wassenaer, Briet, Dekker, and Kok, 2005; Ward \& Beachy, 2003), el bajo peso al nacer (Elgen, Sommerfelt, \& Ellersten, 2003; Mikkoka et al., 2005; Pallás Alonso, 2004), los partos múltiples (van den Oord, Boomsma, \& Verhulst, 2000; Topp, Huusom, Langhoff-Roos, Delhumeau, Hutton, \& Dolk, 2004), etc.

Estas consecuencias en el desarrollo del sujeto se centran, en general, en los aprendizajes escolares, las funciones ejecutivas, el lenguaje y/o el control conductual. En suma, afectan a su potencialidad de adaptación personal, escolar y social. Es tal la seguridad en los resultados que incluso se apunta ya la idea de que los factores de riesgo son buenos predictores de las dificultades cognitivas y personales que se van poniendo en evidencia a lo largo de los años infantiles (Lumley, 2003; Stanton-Chapman, Chapman, Bainbridge, \& Scott, 2002; Sommerfelt et alt. 2000; Sonnander, 2000).

En consecuencia, hay razones contundentes sobre la importancia de la detección del riesgo y de la actuación en él ya que las intervenciones en la primera infancia tienen un efecto perdurable sobre la capacidad cognitiva, la personalidad y la conducta social (Arizcun, 2002; García-Sánchez, Caballero y Castellanos, 1996; McCormick et al., 2006; Perlman, 2001; Poch, Sastre, Merino, Escolano, Farriol y Gil, 2003; Ramos, Marquez y Lluch, 1992).

\section{Método}

\section{Participantes}

Se han recogido los datos correspondientes a los nacimientos desde Enero de 1998 a Diciembre de 2003 que representan el 95\% de los nacimientos en La Rioja, tras autorización para acceder al archivo de los nacimientos habidos en los Hospitales San Millán de Logroño y Fundación Hospital de Calahorra.

\section{Procedimiento}

Del registro de nacimientos, se extraen los niños nacidos con factores de riesgo, sin tener en cuenta los niños fallecidos durante el periodo perinatal. Estos datos, se completan con los recogidos en la Unidad de Seguimiento del Desarrollo postnatal.

El intervalo temporal estudiado posibilita calcular el índice de prevalencia y de incidencia para conocer, no sólo el estado descriptivo de la realidad sino también su tendencia diferencial de ocurrencia de los factores.

Para la clasificación de factores de riesgo se han tenido en cuenta los criterios de la Sociedad Española de Neonatología (2001), también publicados en el Libro Blanco de Atención Temprana (Grupo de Atención Temprana, 2000).

En concreto, los datos que se han tenido en cuenta han sido: a) el tipo de factor de riesgo; b) el momento de la detección e inicio de intervención, en función de lo 
cual se ha clasificado como: b.1) Recién nacido ( $\mathrm{RN}$ ), durante el periodo perinatal; b.2) Detección temprana (DT), entre el primer y sexto mes de vida; b.3) Detección tardía (DTd) después de los seis meses de vida.

Se han extraído, mediante el programa estadístico SPSS, los índices de prevalencia e incidencia para cada tipo de factor en función de su representación en el intervalo estudiado y el momento de la detección.

\section{Resultados}

Tal como puede observarse en la Figura 1, el número de nacimientos en el intervalo temporal entre 1998-2003 en La Rioja, mantiene una tendencia continuada al alza.

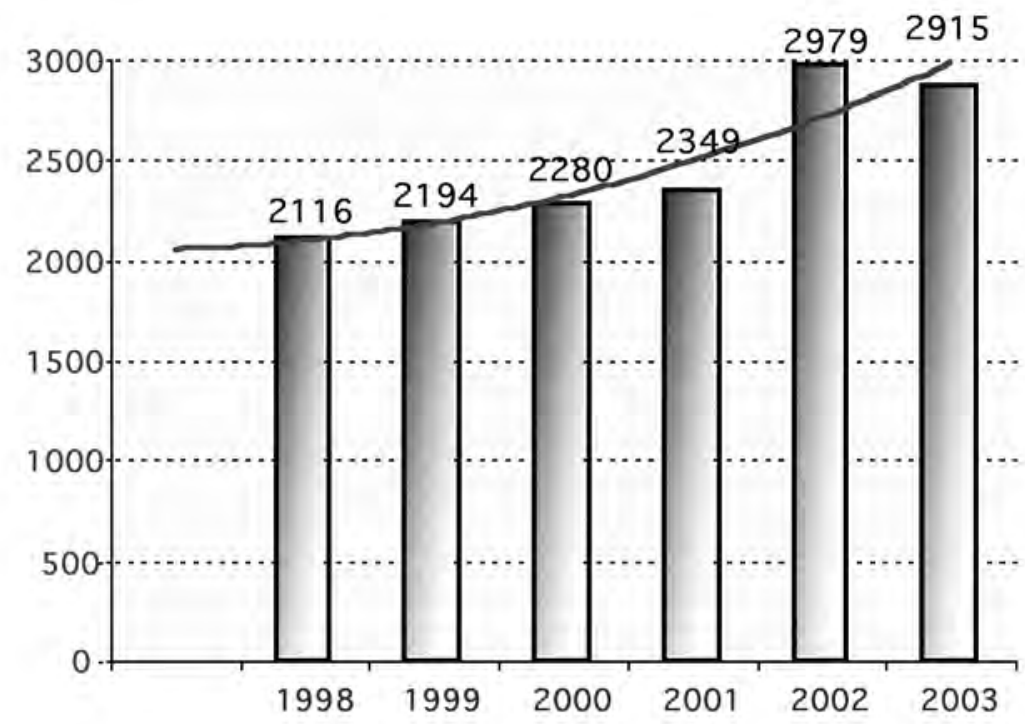

\begin{tabular}{|c|c|c|c|c|c|}
\hline$F$ & +78 & +86 & +69 & +630 & -64 \\
\hline$\%$ & 3,69 & 3,92 & 3,03 & 26,82 & $-2,14$ \\
\hline
\end{tabular}

\begin{tabular}{|c|c|}
\hline Crecimiento total 1998-2003 & 799 \\
\hline$\%$ & 35,73 \\
\hline
\end{tabular}

Figura 1. Nacimientos en La Rioja (1998, 1999, 2000, 2001, 2002, 2003).

En cuanto a la distribución de la frecuencia de nacimientos con riesgo, los datos representados en la Figura 2, muestran una prevalencia que oscila entre 3'38\% y el $4^{\prime} 73 \%$, con una incidencia del $4^{\prime} 07 \%$ entre los recién nacidos, tal como puede observarse en la Figura 3. 


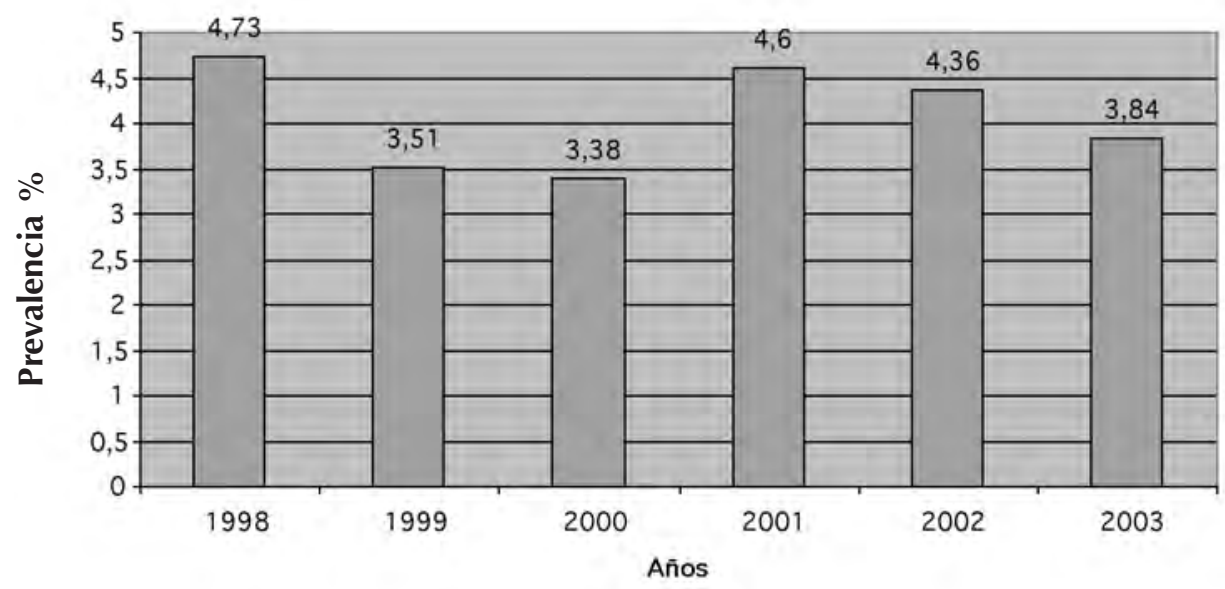

Figura 2. Prevalencia de nacimientos con riesgo respecto al total de nacimientos (1998-2003).

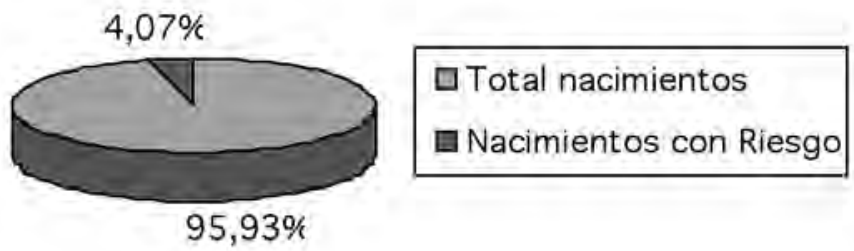

Figura 3. Distribución comparativa de la incidencia de los nacimientos totales y los nacimientos con factores de riesgo (1998-2003).

En la Tabla 1 y en la Figura 4, se representan los factores de riesgo de mayor prevalencia e incidencia, respecto al total de nacimientos habidos. Dada la amplitud de factores de riesgo se recogen, solamente, aquellos con mayor incidencia: a) la prematuridad $\left(2^{\prime} 01 \%\right)$, entendiendo como tal al recién nacido por debajo de 36 semanas de gestación; b) las asimetrías $\left(0^{\prime} 50 \%\right)$ diagnosticadas ante hematomas del esternocleidomastoideo, las craneo-faciales y las contracturas congénitas de cadera; c) el retraso de desarrollo madurativo $\left(0^{\prime} 30 \%\right)$; d) el bajo peso para la edad gestacional (C.I.R.), con un peso inferior al P10 respecto a la Edad gestacional $\left(0^{\prime} 26 \%\right)$; y, e) la anoxia perinatal $\left(0^{\prime} 24 \%\right)$ que comprende a los recién nacidos con Apgar menor de 3 al minuto o menor de 7 a los 5 minutos. 
Tabla 1. Factores de riesgo de mayor prevalencia e incidencia respecto a la población nacida (1998-2003).

\begin{tabular}{|l|c|c|c|c|c|c|c|}
\hline Años & 1998 & 1999 & 2000 & 2001 & 2002 & 2003 & $\Sigma$ \\
\hline Nacimientos & $\mathrm{n}=2116$ & $\mathrm{n}=2194$ & $\mathrm{n}=2280$ & $\mathrm{n}=2349$ & $\mathrm{n}=2979$ & $\mathrm{n}=2915$ & $\mathrm{~N}=14833$ \\
\hline Factores de riesgo & Prevalencia & Prevalencia & Prevalencia & Prevalencia & Prevalencia & Prevalencia & Incidencia \\
\hline Prematuridad & 2,60 & 1,23 & 1,75 & 2,26 & 2,28 & 1,89 & 2,01 \\
\hline Asimetrías & 0,52 & 0,73 & 0,31 & 0,64 & 0,37 & 0,48 & 0,50 \\
\hline Retraso del desarrollo & 0,28 & 0,50 & 0,61 & 0,30 & 0,13 & 0,07 & 0,30 \\
\hline Bajo peso & 0,05 & 0,00 & 0,18 & 0,38 & 0,54 & 0,31 & 0,26 \\
\hline Anoxia perinatal & 0,47 & 0,23 & 0,13 & 0,21 & 0,10 & 0,31 & 0,24 \\
\hline
\end{tabular}
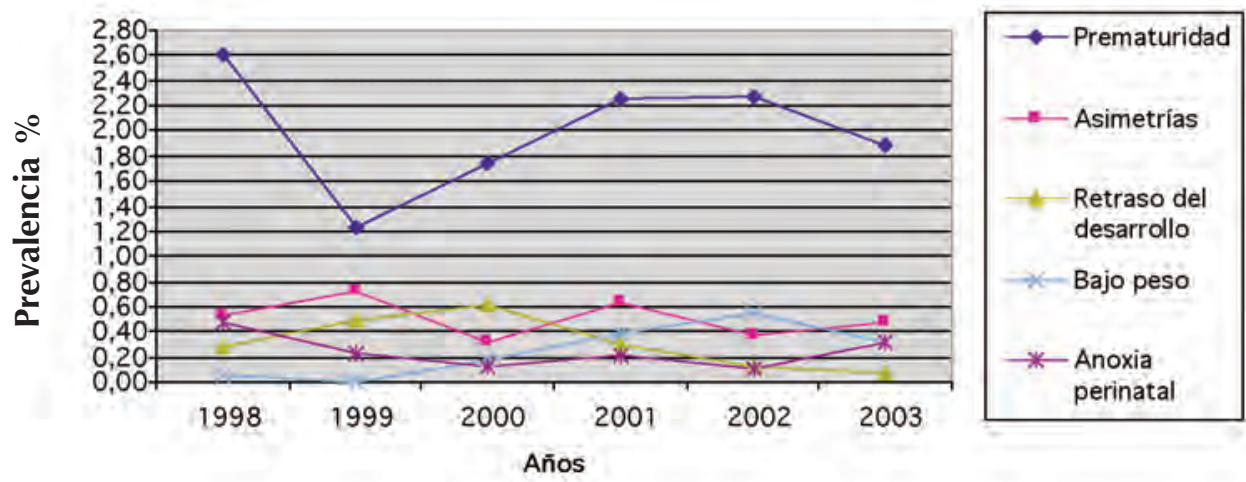

Figura 4. Prevalencia de los factores de riesgo respecto a la población nacida (1998-2003).

Los resultados correspondientes a la prevalencia e incidencia de los factores de riesgo, respecto a la propia población de riesgo, se recogen en la Tabla 2 y en la Figura 5. El factor con mayor incidencia es la prematuridad $\left(49^{\prime} 42 \%\right)$, seguido por: la asimetría postural (12'27\%); el retraso de desarrollo de origen desconocido (7'13\%); el bajo peso al nacer (CIR) (6'47\%); y, la anoxia perinatal (5'79\%). 
Tabla 2. Factores de riesgo de mayor prevalencia e incidencia respecto a la población nacida con riesgo (1998-2003).

\begin{tabular}{|l|c|c|c|c|c|c|c|}
\hline Años & 1998 & 1999 & 2000 & 2001 & 2002 & $2003 \quad \Sigma$ & \\
\hline Nacimientos & $\mathrm{n}=100$ & $\mathrm{n}=77$ & $\mathrm{n}=77$ & $\mathrm{n}=108$ & $\mathrm{n}=130$ & $\mathrm{n}=112$ & $\mathrm{~N}=604$ \\
\hline Factores de riesgo & Prevalencia & Prevalencia & Prevalencia & Prevalencia & Prevalencia & Prevalencia & Incidencia \\
\hline Prematuridad & 55,0 & 35,1 & 51,9 & 49,1 & 52,3 & 49,1 & 49,34 \\
\hline Asimetrías & 11,0 & 20,8 & 9,1 & 13,9 & 8,5 & 12,5 & 12,25 \\
\hline Retraso del desarrollo & 6,0 & 14,3 & 18,2 & 6,5 & 3,1 & 1,8 & 7,28 \\
\hline Bajo peso & 1,0 & 0,0 & 5,2 & 8,3 & 12,3 & 8,0 & 6,46 \\
\hline Anoxia perinatal & 10,0 & 6,5 & 3,9 & 4,6 & 2,3 & 8,0 & 5,79 \\
\hline
\end{tabular}

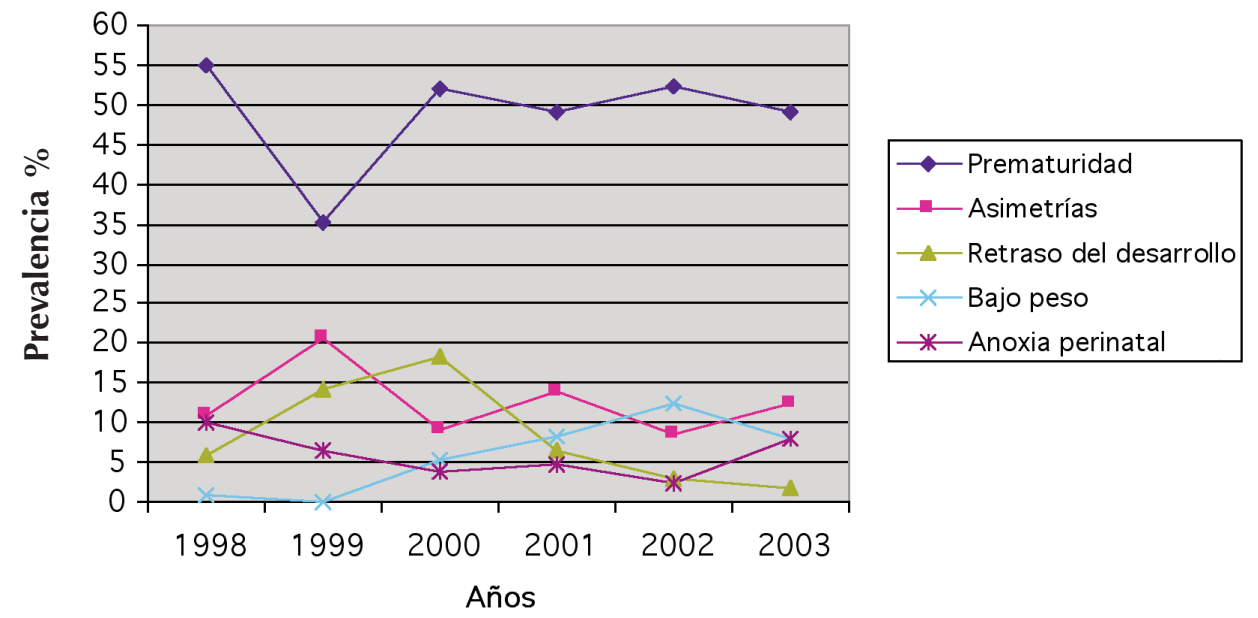

Figura 5. Prevalencia de los factores de riesgo respecto a la población nacida con riesgo (1998-2003).

Finalmente, respecto al momento de la detección del riesgo y la puesta en marcha del programa de seguimiento e intervención, la Figura 6, representa el porcentaje de niños detectados en cada uno de los períodos estudiados: nacimiento, detección temprana (antes de los 6 meses) y detección tardía (después de los 6 meses de vida). 


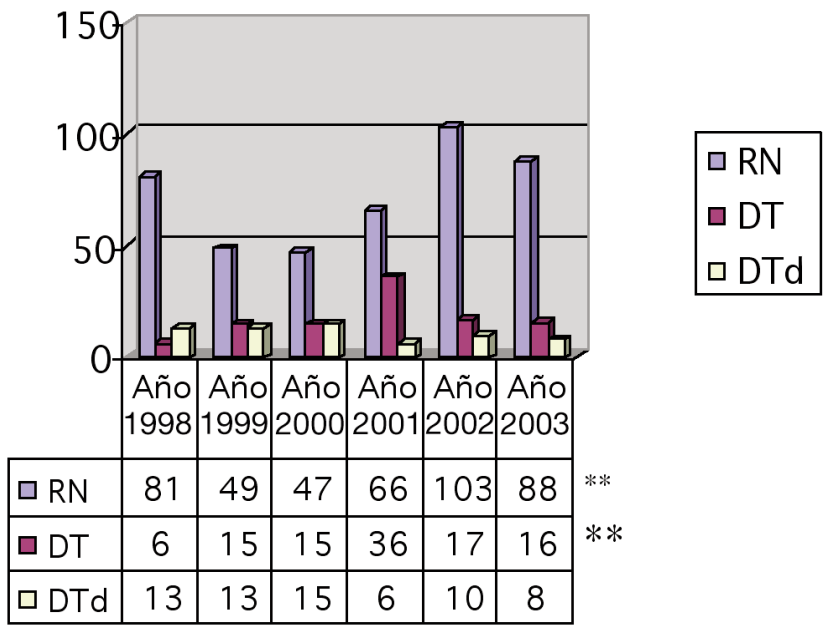

Figura 6. Momentos de detección de riesgo: (1998-2003).

Dónde: ${ }^{* *}=$ Diferencias significativas $p \leq .001$

Tal como puede observarse, hay diferencias significativas interanuales en el nivel de detección realizada en edades tempranas: perinatal $(p>.0001)$ y antes de los 6 meses ( $p>$.0001), con una tendencia hacia el incremento. En cambio, no se obtienen diferencias significativas respecto al nivel de detección tardío $(p=.3656)$.

\section{Discusión}

A nivel global, los resultados obtenidos, relativos al índice de natalidad en la Comunidad Autónoma de La Rioja, son acordes con los nacionales, mostrando una tendencia sostenida al incremento, desde el año 1997 hasta el 2003 (inclusives).

Por otra parte, la prevalencia e incidencia de nacimientos con riesgo en la Comunidad Autónoma estudiada, son próximas a la cifra que estima la OMS (2006) y el Programa Helios (1996) a nivel europeo, situada alrededor del 5\%.

Entre los factores de riesgo detectados, la prematuridad y el crecimiento intrauterino retardado, son los factores más frecuentes. Esta tendencia parece ser generalizada (Della Piazza, 1997; Sommerfelt et al., 2000; Sonnader, 2000). El índice mundial de niños nacidos con bajo peso, propuesto por la OMS (2006) es el del 17\%. Según Arizcun (2002) entre los niños nacidos en situación de riesgo (3'2\%) en nuestro país, los factores que más frecuentemente se presentan son: la prematuridad y peso por debajo de 1500gr; otros factores ordenados según su frecuencia son: la ventilación artificial durante más de 24 horas, el crecimiento intrauterino retardado, la anoxia perinatal, sepsis y meningitis neonatales, hemorragias cerebrales, hijos de madres drogadictas o alcohólicas y la afectación neurológica neonatal. En nuestro estudio hemos encontrado resultados similares en cuanto a las sepsis y meningitis y a la afectación neurológica neonatal. 
Dada la extensión de los factores de riesgo encontrados, se han expuesto en este trabajo sólo los más relevantes. Queremos llamar la atención sobre dos factores de riesgo de menor incidencia y prevalencia, aunque de importante influencia en el desarrollo, como son el hipotiroidismo congénito y las cardiopatías congénitas con repercusión hemodinámica.

Es interesante resaltar que los resultados muestran un importante número de niños con factores de riesgo detectados tempranamente, lo cual puede facilitar el establecimiento de programas de seguimiento y de intervención eficaces para prevenir u optimizar su curso de desarrollo (Arizcun, 2002; Escobar, Littenberg, \& Petiti, 1991; García-Sánchez, Caballero y Castellanos, 1996; Grupo de Atención Temprana, 2000; Guralnick 1993; Poch, Sastre, Merino, Escolano, Farriol y Gil, 2003).

En este sentido, la intervención temprana está facilitada por la ubicación de la Unidad de Seguimiento en el mismo Hospital de referencia como Unidad Neonatal, a esto se le suma la colaboración con los pediatras de Atención Primaria.

Ante la mejora de la atención en las Unidades Neonatales, la mayor supervivencia de niños de riesgo plantea el reto de asegurar un sistema que lleve a cabo un plan de servicios que responda a las necesidades del niño y su familia mediante evaluaciones y actuaciones periódicas en el curso del desarrollo de todos los niños con riesgo. Las razones de la importancia de la detección del riesgo y de la actuación en él ya se han planteado en la introducción de este trabajo, dado que las intervenciones en la primera infancia tienen un efecto perdurable sobre la capacidad cognitiva, la personalidad y la conducta social del ser humano (Arizcun, 2002; Hill, BrooksGunn, \& Waldfogel, 2003; Doyle, 2001; Gardner, Walker, Powell, GranthamMcGregor, 2003; Olds, 2006; Perlman, 2001; Ramos, Marquez y Lluch, 1992).

\section{Bibliografía}

Arizcun, J. (2002). Aspectos neonatológicos y factores de riesgo en atención temprana. Revista de Neurología, 34(1), 136-182.

Blackman, J. A. (2002). Early Intervention: A Global Perspective. Infants \& Young Children, 15(2), 11-19.

Cambrodí, A. (1983). Principios de Psicología Evolutiva del Deficiente Mental. Barcelona: Herder.

De Haan, M. \& Johnson, M. (2003). The cognitive Neurosicence of Development. New York, NY: Psychology Press.

Della Piazza, S. (1997). L'enfant prematuré: le point sur la question. Bruxelles: De Boeck Université.

Diamond, A. (2002). Normal development of prefrontal cortex from birth to young adulthood: Cognitive functions, anatomy and biochemistry. En D.T. Stuss \& R.T. Knighte (Eds.), Principles of Frontal Lobe Function (pp.466-503). London: Oxford University Press.

Doyle, L. (2001). Outcome at 5 years of age of children 23 to 27 weeks'gestation: refining the prognosis. Pediatrics, 108(1), 134-141.

Elgen, I., Sommerfelt, K., Ellersten, B. (2003). Cognitive performance in a low birth weight cohort at 5 and 11 years of age. Pediatric Neurology, 28(3), 1-6. 
Elster, N. (2000). Less is more: the risk of multiple births. Fertility and Esterility, 74(4), 617-623.

Escobar, G. J., Littenberg, B., Petiti, D. B. (1991). Outcome among surviving very low birth weight infants: A meta-analysis. Archives of Disease in Childhood, 66, 204211.

Farley, T. A., Mason, K., Rice, J., Habel, J. D. , Scribner, R. and Cohen, D. A. (2006). The relationship between the neighbourhood environment and adverse birth outcomes. Paediatric \& Perinatal Epidemiology, 20(3), 188-200.

Figueras Aloy, J. (2004). Coordinación interdisciplinaria de la atención al recién nacido de alto riesgo. Propuestas de actuación. Boletín de Pediatría, 44, 182-184.

García-Sánchez, F.A., Caballero, P.A. y Castellanos, P. (1996). Características físicas y psicomotoras, modalidades de tratamiento y escolarización en distintos tipos de parálisis cerebral infantil en la región de Murcia. Anales de Pedagogía, 12, 175 193.

Gardner, J. M., Walker, S. P., Powell, C. A., \& Grantham-McGregor, S. (2003). A randomized controlled trial of a home-visiting intervention on cognition and behavior in term low birth weight infants. Journal of Pediatrics, 143(5), 634-639.

Guralnick, M. J. (1993). Second generation research on the effectiveness of early intervention. Early Education and Development, 4, 366-378.

Grupo de Atención Temprana (2000). Libro blanco de la Atención Temprana. Madrid: Real Patronato de Prevención y de Atención a Personas con Minusvalía. Consultado el 25 de Mayo, 2006, en http://paidos.rediris.es/gat/2000.htm

Hill, J. L., Brooks-Gunn, J., \& Waldfogel, J. (2003). Sustained effects of high participation in an early intervention for low-birth-weight premature infants. Developmental Psychology, 39(4), 730-44.

Karmiloff-Smith, A. (1997). Promissory notes, genetic clocks, and epigenetic outcomes. Behavioral and Brain Sciences, 20(2), 355-360.

Lumley, J. (2003). Defining the problem: the epidemiology of preterm birth. BJOG: an International Journal and Obstetrics and Gynaecology, 110(20), 3-7.

McCormick, M. C., Brooks-Gunn, J., Buka, S. L., Goldman, J., Yu, J., Salganik, M., Scott, D. T., Bennett, F. C., Kay, L.L., Bernbaum, J. C., Bauer, C. R., Martin, C., Woods, E. R., Martin, A., \& Casey, P. H. (2006). Early intervention in low birth weight premature infants: results at 18 years of age for the Infant Health and Development Program. Pediatrics, 117(3), 771-780.

Mellier, D., \& Fessard, C. (1998). Preterm birth and cognitive inhibition. European Review of Applied Psychology, 48, 13-18.

Mikkola, K., Ritari, N., Tommiska, V., Salokorpi, T., Lehtonen, L., Tammela, O., Paakkonen, L., Olsen, P., Korkman, M., \& Fellman, V. (2005). Neurodevelop-mental outcome at 5 years of age of a national cohort of extremely low birth weight infants who were born in 1996-1997. Pediatrics, 116(6), 1391-1400.

Mounoud, P. (1996). Perspective taking and belief attribution. From Piaget's theory to children's theory of mind. Swiss Journal of Psychology. 55(2), 93-103.

Munakata, Y., Casey, B. J., \& Diamond, A. (2004). Developmental cognitive neuroscience: progress and potential. TRENDS in Cognitive Sciences, 8(3), 122-128. 
Olds, D. (2006). Progress in Improving the Development of Low Birth Weight Newborns. Pediatrics, 117(3), 940-941.

Organización Mundial de la Salud (2006). Informe sobre la salud en el mundo. Consultado el 24 de Mayo, 2006, en http://www.who.int/whr/es/index.html

Pallás Alonso, C.R. (2004). El recién nacido menor de 1.500 g y la discapacidad. Boletín de Pediatría, 44, 193-197.

Perlman, J. (2001). Neurobehavioral deficits in premature graduates of intensive care potential medical and neonatal environmental risk factors. Pediatrics, 108 (6), 1339-1347.

Plomin, R., \& Petrill, S. (1997). Genetics and intelligence: What's new? Intelligence, $24(1), 53-77$.

Poch, M. L., Sastre, S., Merino, N., Escolano, E., Farriol, R., y Gil, J. (2003). Unidades de seguimiento del desarrollo. Revista de Atención Temprana, 6(1), 23-28.

Programa Helios I (1996). Intervention precoce. Information, orientation et guidance des famillas. Consultado el 25 de Mayo, 2006, en http://paidos.rediris.es/genysi/recursos/doc/leyes/guia_bpr.htm

Programa Helios II (1996). Prevención, detección temprana, intervención e integración. Consultado el 25 de Mayo, 2006, en http://paidos.rediris.es/genysi/recursos/doc/leyes/heliosii.htm

Quartz, S. R. \& Sejnowski, T. J. (1997). The neural basis of cognitive development: A constructivist manifest. Behavioral and Brain Sciences, 20, 537-596.

Ramos, I., Marquez, A. y Lluch, M. D. (1992). Control psiconeuroevolutivo de recién nacidos de alto riesgo. Anales Españoles de Pediatría, 35, 313-318.

Sastre, S. y Poch, M. L. (2001). Prevención e intervención en el desarrollo cognitivo Temprano. Revista de Salud y Servicios Sociales, 64, 9-11.

Sociedad Española de Neonatología (2001). Recomendaciones de mínimos para la asistencia del recién nacido sano. Consultado el 25 de Mayo, 2006, en http://www.se-neonatal.es/

Sommerfelt, K., Andersson, H. W., Sonnander, K., Ahlsten, G., Ellertsen, B., Markestad, T., Jacobsen, G., Hoffman, H. J. and Bakketeig, L. (2000). Cognitive development of term small for gestational age children at five years of age. Archives of Disease in Childhood, 83, 25-30.

Sonnander, K. (2000). Early identification of developmental disabilities. Acta Paediatrica, 89(Suppl. 434), 17-23.

Stanton-Chapman, T. L., Chapman, D. A., Bainbridge, N. L., \& Scott, K. G. (2002). Identification of early risk.factors for language impairment. Research in Developmental Dissabilities, 23(6), 390-405.

Thompson, J. M. D., Irgens, L. M., Rasmussen, S., Daltveit, A. K. (2006). Secular trends in socio-economic status and the implications for preterm birth. Paediatric and Perinatal Epidemiology, 20(3), 182-187.

Topp, M., Huusom, L. D., Langhoff-Roos, J., Delhumeau, C., Hutton, J. L., \& Dolk, H. (2004). Multiple birth and cerebral palsy in Europe: a multicenter study. Acta Obstetricia et Gynecologica Scandinavica, 83(6), 548-53. 
Valle Trapero, M. (2002). Intervención precoz en niños de alto riesgo biológico. Tesis Doctoral. Universidad Complutense de Madrid. Consultado el 25 de Mayo, 2006, en http://www.ucm.es/eprints/2236/

Valle Trapero, M. (1999). La función de la familia en el niño hospitalizado. Revista Latinoamericana de Neonatología, 1, 111-113.

van Baar, A. L., van Wassenaer, A. G., Briet, J. M., Dekker, F. W., Kok, \& J. H. (2005). Very Preterm Birth is Associated with Disabilities in Multiple Developmental Domains. Journal of Pediatric Psychology, 30(3), 247 - 255.

van den Oord, E. J.; Boomsma, D. I., \& Verhulst, F. C. (2000). Study of Genetic and Environmental Effects on the Co-Occurrence of Problem Behaviors in Three-YearOld Twins. Journal of Abnormal Psychology, 109, 360-372.

Ward, R. M, \& Beachy, J. C. (2003). Neonatal complications following preterm birth. BJOG: an International Journal of Obstetrics and Gynaecology, 110 (20), 8-16.

Wolke D. (2005). Desarrollo psicológico a largo plazo de los niños muy pretérmino y otros de riesgo neonatal. Consultado el 25 de Mayo, 2006, en http://paidos.rediris.es/genysi/recursos.htm 
